\title{
THE USE OF FIBRINOLYTIC ACTIVATORS IN MENINGITIS AND SIMILAR CONDITIONS
}

\author{
BY \\ R. L. NEWMAN and G. T. STEWART \\ From the Department of Pathology, Queen Mary's Hospital for Children, Carshalton, Surrey
}

(RECEIVED FOR PUBLICATION AUGUST 24, 1964)

The deposition of fibrin in the ventricles and subarachnoid cisternae of the brain, which occurs in bacterial meningitis, seldom causes complication in patients who receive adequate chemotherapy, provided the causal organism is quickly eliminated and no anatomical abnormality is present. Fibrin deposition still occurs, however, in certain circumstances, e.g. in children with congenital abnormalities of the central nervous system and after neurosurgical procedures with or without infection. In these cases, infections are often due to staphylococci or Gram-negative bacilli, particularly Proteus and $P s$. pyocyanea, which are relatively resistant to antibiotics. When infection persists, the protein content of the cerebrospinal fluid (CSF) increases to very high levels. Deposition of fibrin from the CSF can then occur leading to loculation, hydrocephalus, and additional difficulties in surgical treatment. It is of interest, therefore, to investigate the possibility of using fibrinolytic agents in preventing the deposition of fibrin in such cases and in the lysis of fibrin already deposited.

The lysis of intravascular thrombi can now be achieved in certain cases by activation of the physiological plasma fibrinolytic system. It has been shown that thrombi contain a high concentration of plasminogen (Blombäck and Blombäck, 1956) and that activator in the surrounding medium diffuses readily into established clots (Alkjaersig, Fletcher, and Sherry, 1959). It is generally agreed that the lysis of intravascular thrombi is, therefore, best achieved by administration of activators of plasminogen such as streptokinase and urokinase, thereby producing a high level of plasmin within the clot itself, without a hyperplasminaemic state in the plasma (Sherry, Lindemeyer, Fletcher, and Alkjaersig, 1959). These considerations would presumably apply also to established fibrin deposits in the subarachnoid and ventricular spaces, but in aiming at the prevention of fibrin formation it would be advantageous to produce a high level of plasmin in the CSF.

Cathie (1949) reported the use of a streptokinase preparation in tuberculous meningitis. The preparations in use at that time, however, were relatively crude and contained many impurities so that serious reactions were often provoked. Fletcher (1954) using a streptokinase-streptodornase preparation concluded that this might be used to induce an effective level of fibrinolytic activity in certain cases, but found some reactions to larger doses and suggested that inhibitors might also be present.

The first necessity when considering treatment of this kind is to demonstrate the components of the fibrinolytic system in the CSF, that is, to ascertain that plasminogen is present and amenable to activation and that inhibitors if present can be neutralized. To investigate these fundamentals, specimens of CSF from cases of meningitis and from post-meningitis cases were examined, together with as many normal specimens as possible, by the techniques described below.

\section{Preliminary Laboratory Studies}

Generally speaking, existing methods for assay of components of the fibrinolytic system are unsuitable for use with CSF, being too insensitive or too time consuming for this purpose. New methods or modifications of existing ones have therefore been devised.

Plasminogen. A new method for plasminogen assay was used which has a high degree of sensitivity and is suitable for detection and measurement of plasminogen at low levels (Newman, 1964a). This is a fibrinolytic assay using a standardized bovine fibrin clot. Streptokinase is added to the clot mixture and the lysis time is predictable with known quantities of plasminogen. Plasminogen is supplied by the CSF being tested, and the lysis time depends on the amount of plasminogen present. Lysis times are read visually or by means of a photoelectric instrument (Newman, 1964b). A standard curve can be drawn relating these co-ordinates, which correlates 


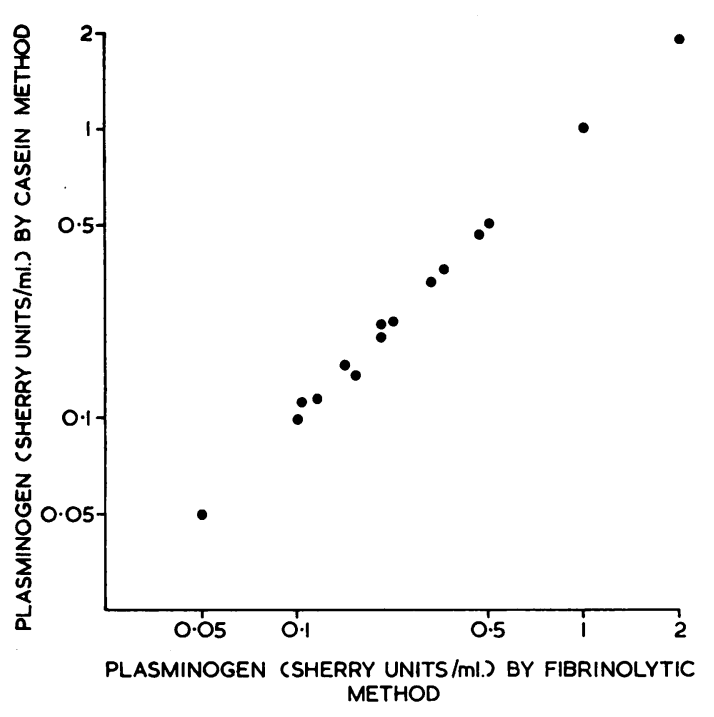

FIG. 1.-Comparison of fibrinolytic and caseinolytic methods of plasminogen assay.

closely with values obtained by caseinolytic assay (Fig. 1).

Activator. A modification of the above method proved suitable for detection of activator in small amounts in CSF. This consists of the omission of streptokinase from the clot mixture and enrichment of the clot with added human plasminogen. Lysis time then depends on the

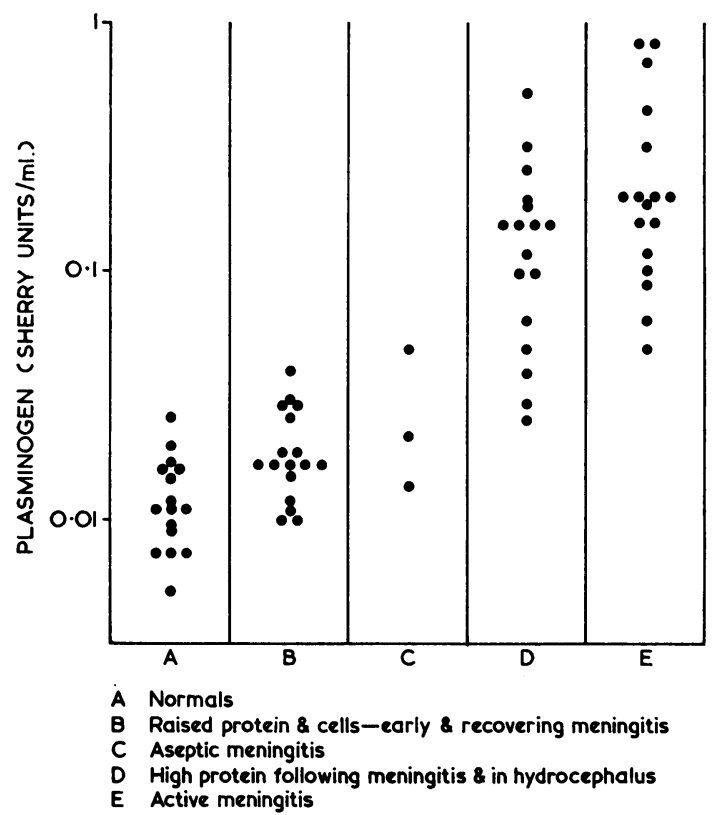

FIG. 2.-Plasminogen content of normal and abnormal CSF. amount of activator present, as in the method described below for detection of antibodies.

Antibodies. A method was also devised for detection and measurement of anti-streptokinase (Newman, 1965). In this, streptokinase is added to a plasminogen-enriched bovine fibrin clot. Anti-streptokinase is demonstrated quantitatively by alteration of the lysis time caused by addition of CSF to the system.

Seventy-five specimens were examined for plasminogen level and for the presence of activator. Antibody tests were conducted with a few normal specimens and specimens from patients under treatment with activator.

\section{Results}

Plasminogen. All specimens of CSF examined, normal and abnormal, contained plasminogen in varying amount. In normal specimens trace amounts only were found, never exceeding 0.026 Sherry units per ml., which is about $1 / 100$ of the normal plasma level. In nearly all the pathological specimens examined higher levels of plasminogen

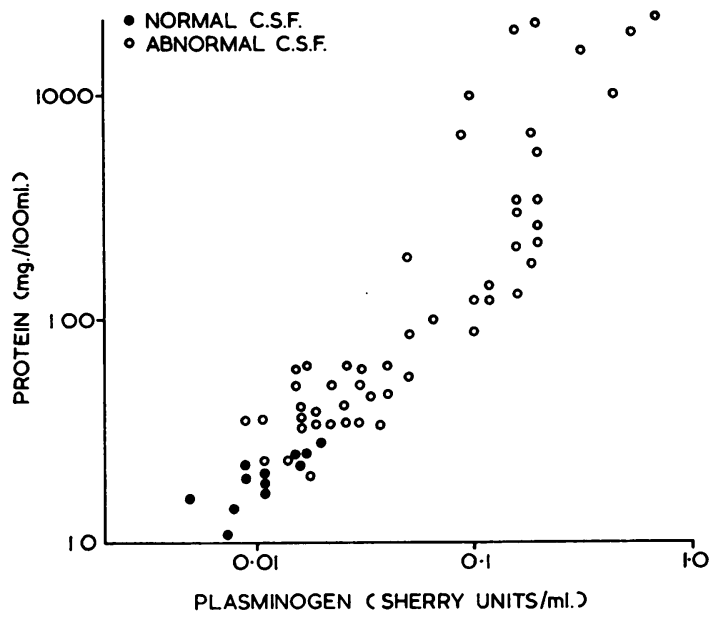

FIG. 3.-Relation between plasminogen and protein in CSF.

were found (Fig. 2). The highest levels occurred in the acute stage of pyogenic meningitis, during the first few days of the disease when organisms were cultured from the CSF and the protein was increased. In very early cases of meningitis (within the first 24 hours of the disease), when the protein was only slightly increased but the cell count raised, smaller increases were found, as also in a few cases of aseptic meningitis or encephalitis with raised lymphocyte counts in the CSF and increased protein. High levels were also found in cases of chronic hydrocephalus where the protein level was raised.

There was fairly close correlation between plasminogen and protein levels in the CSF (Fig. 3). 
Paper electrophoresis at $p \mathrm{H} 8.6$ of specimens with high protein levels showed patterns similar to the plasma proteins but with relatively less $\alpha_{1}$ - and $\gamma$-globulin and relatively more $\beta$-globulin. Fibrinogen was inconstantly present. After dilution of plasma so that the protein concentration was commensurate with that in the CSF, and electrophoresis as above, comparable zones of lysis appeared around the $\beta$-globulin bands of both fluids when the strips were left overnight on bovine fibrin plates containing streptokinase (Fig. 4). This confirmed the presence of the estimated amount of plasminogen in the $\beta$-globulin band in the CSF. In cases of meningitis where serial CSF specimens were obtained early in the illness, plasminogen rose rapidly to high levels, sometimes within the first 48 hours. The rate of rise in plasminogen was faster than the rate of rise in protein, which sometimes continued to rise thereafter with no further increase in plasminogen.

Activator. Of 72 specimens examined, normal and abnormal, no activator was detectable in any of them by the method used.

Inhibitors. Not all specimens were examined for inhibitors as the method was developed recently. In those tested no anti-streptokinase was detected.

It is apparent from these results that plasminogen is present in quantity in the CSF in inflammatory and post-inflammatory states, no intrinsic activator is present, and inhibitors are not detectable. The plasminogen is capable of activation in vitro by streptokinase and urokinase, and fibrin clots incorporating such CSF in small quantities are lysed by addition of activator. It seems likely, therefore, that intrathecal or intraventricular administration of activators might produce a fibrinolytic state in the CSF.

\section{Clinical Studies}

The laboratory results described above provide the basis of an exploratory clinical trial in selected patients in whom abnormal deposition of fibrin appeared to be a factor in preventing recovery from meningitis and ventriculitis.

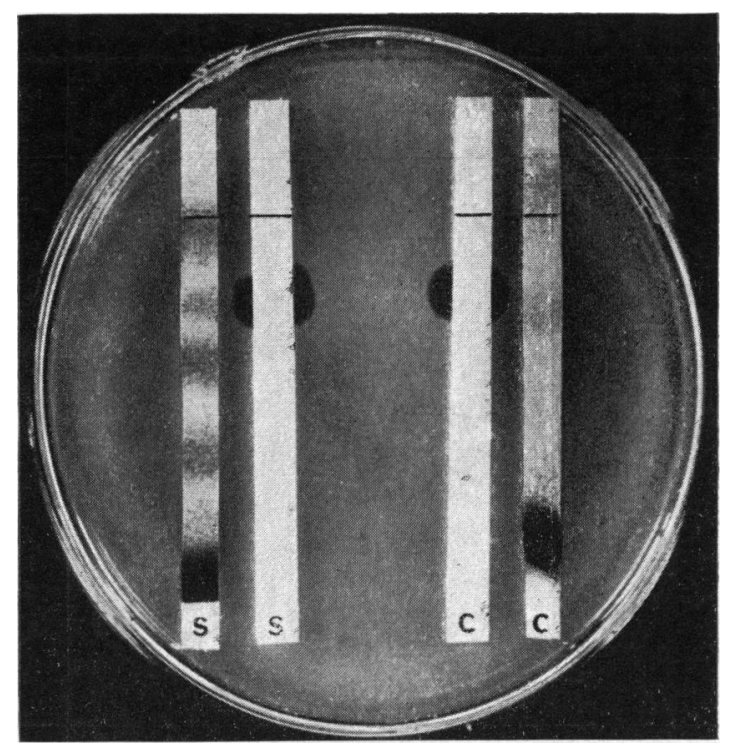

FIG. 4.-Fibrin plate showing comparably the plasminogen content in CSF and diluted serum.

Selection of Cases. Seventeen patients were selected for fibrinolytic treatment; of these 16 were babies and one was a child of 5 years (Table 1). Of the 16 babies, 12 were cases of meningitis arising before, or immediately after, repair of a meningomyelocele. In 7 of these the infecting organism was Ps. pyocyanea and in 4 it was Proteus. In the remaining one the organism was a Gram-negative bacillus which could not be cultured; 2 cases developed subarachnoid blocks during the course of acute coccal meningitis (1 Staph. aureus, 1 Meningococcus) being treated with antibiotics. Two were cases of ventriculitis and meningitis associated with colonization of Spitz-Holter valves, inserted for relief of congenital hydrocephalus. The older child had a large acrocephalic brain hernia, previously repaired, and developed meningitis due to Ps. pyocyanea following a further attempt at surgical repair.

Seven cases were given streptokinase (Kabi) and 10 were given urokinase (Leo). The drug was not selected with any attempt in mind to compare the effects of the two. In most cases it was administered together with the appropriate antibiotic by the intraventricular route,

TABle 1

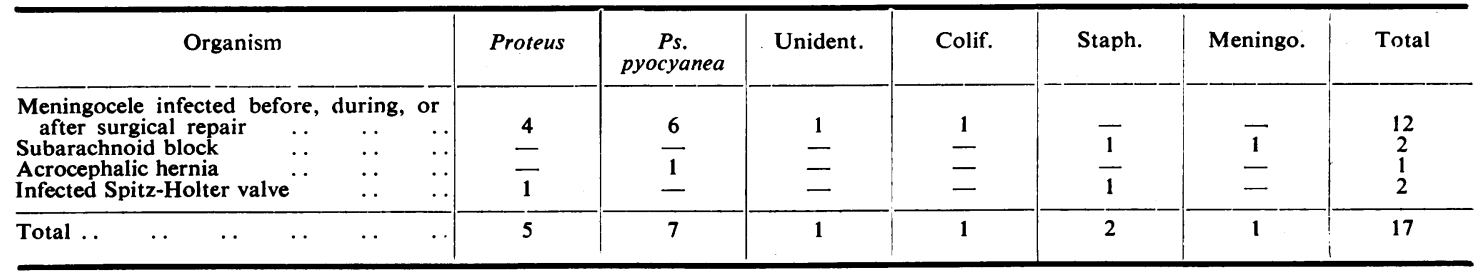




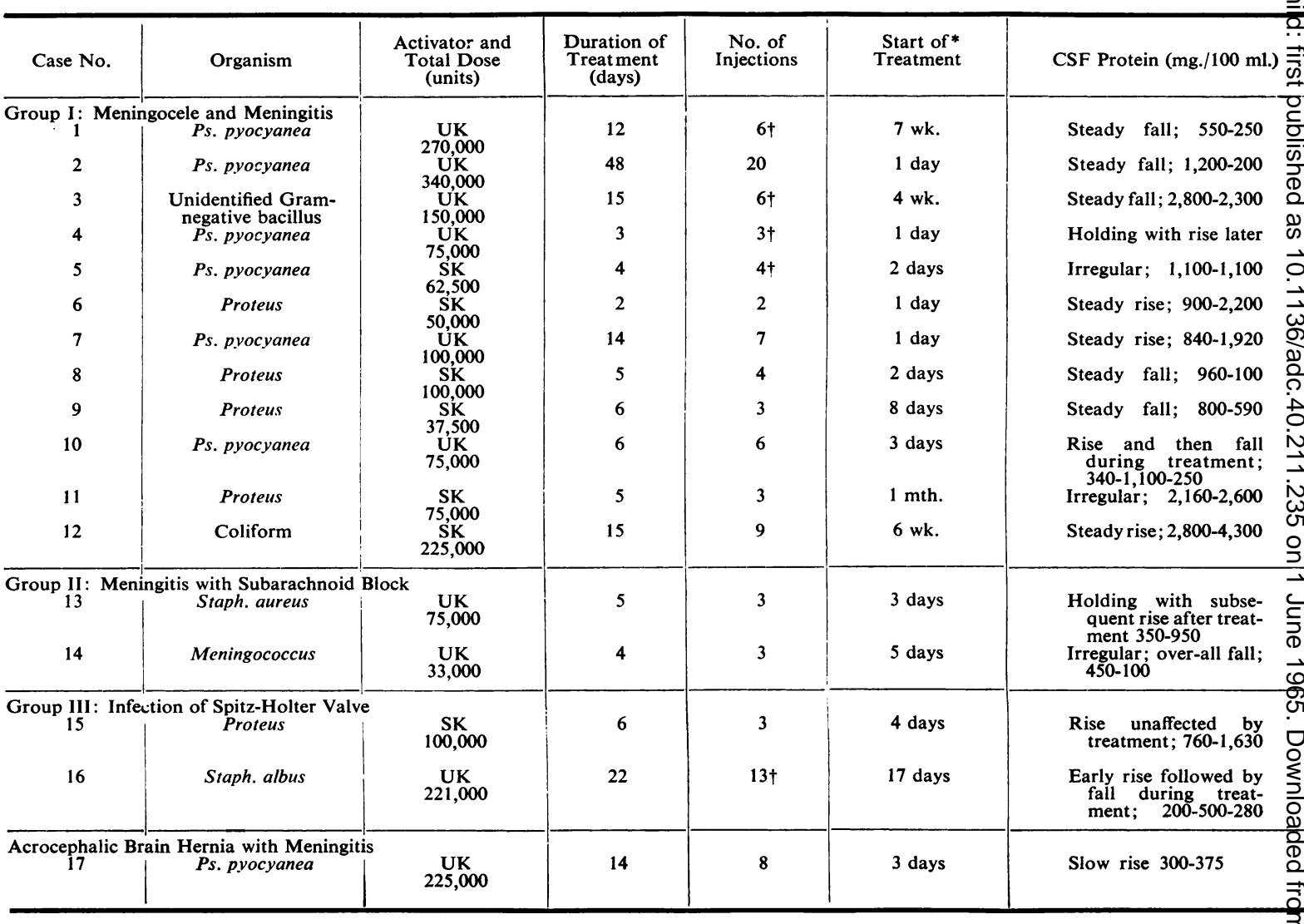

* Interval between onset of meningitis and beginning of fibrinolytic treatment.

UK = Urokinase; SK = streptokinase.

$\uparrow$ EEG during injectio

alternate doses into alternate lateral ventricles, usually daily or on alternate days and for varying periods (Table 2). In one of the cases of subarachnoid block the drug was given by lumbar puncture. Injections consisted of the appropriate dose of activator dissolved in $1 \mathrm{ml}$. of sterile water and in cases where this was combined with an antibiotic the total volume of injection was usually 2 to $2.5 \mathrm{ml}$. which was given after withdrawal of a sample of CSF for examination. This enabled laboratory tests to be made of the state of the CSF before each injection, which was normally 24 or 48 hours after the previous one. In every case a sample of CSF was examined before treatment started, to determine dosage and to obtain a base-line. In two cases treated early in the series protamine sulphate was added to the mixture to prolong the effect of the activator. This, however, appeared to make little difference to the clinical or biochemical results and was later omitted.

Each patient was observed carefully to ascertain whether administration of activator in this way had any toxic or other side-effects. CSF specimens were examined for evidence of meningeal irritation such as rise in protein, pleocytosis, eosinophilia, etc. In 4 cases, EEG recordings were taken before, during, and after the injections and in most other cases within 24 hours of the injections. Duration of treatment varied considerably, from 2 to 20 injections over 2 to 48 days. This depended largely on clinical progress and the results of the laboratory tests. Total dose of urokinase ranged from 33,000 to 340,000 Ploug units and of streptokinase from 37,500 to 225,000 Kabi units. Treatment with activators was sometimes delayed for varying periods in the early cases but, as a result of experience gained, the later cases were given treatment as early as possible, normally with the first antibiotic dose. In some cases activator was given later in the course of the disease after the CSF was sterilized to see if the CSF protein could be lowered.

All specimens obtained during the course of treatment were examined for protein and cells and were cultured routinely. Plasminogen and activator levels were also estimated on all specimens except those contaminated with blood which made the tests difficult to interpret. Inhibitor levels were also estimated in the later cases being treated with streptokinase. 
Died of acute hydrocephalus during treatment

CSF sterilized; early hydrocephalus arrested temporarily

Head diameter increased steadily

CSF sterilized; intracranial pressure fell; head diam. remained static

Died during treatment of acute hydrocephalus

CSF sterilized; hydrocephalus arrested

CSF sterilized but protein continued to rise

CSF sterilized

Hydrocephalus arrested temporarily

Recurrence of meningitis with resistant organism

CSF sterilized; hydrocephalus established 4 wk., no improvement ncreasing hydrocephalus arrested;

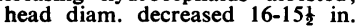

Block relieved after 2nd dose

Block relieved after 1st dose

Infection not cleared

Congenital hydrocephalus; no change

No change

\section{Results}

-

$+$

Good progress; S-H valve; well after

Died of acute hydrocephalus Died in another hospital; no
hydrocephalus

Discharged with arrested hydrocephalus

Died of acute hydrocephalus

Died of acute hydrocephalus

Good progress after S-H valve; well $6 \mathrm{mth}$. later

Died of bronchopneumonia

Died of hydrocephalus

Valve inserted; discharged well
Necropsy

Ventricular walls clear; choroid plexus

buried in fibrin (collagen ++ )

Choroid buried in pyogenic membrane

No necropsy

No necropsy

Choroid buried in fibrin; ventriculitis

No fibrin in ventricles; aqueduct closed

No necropsy

Choroid buried in fibrin (collagen ++ )

Abscesses in both hemispheres; pus in lateral ventricles; aqueduct blocked by old inflammation
Poor progress due to brain damage:
died from

Good recovery; well 5/12 later

Valve replaced; hydrocephalus controlled; well $6 \mathrm{mth}$. later; infection cleared spontaneously, later

Hydrocephalus controlled by valve; died of bronchopneumonia

\section{Laboratory.}

\section{Results}

Plasminogen. All the cases treated showed high levels of plasminogen in the CSF before treatment. None showed any activator and, in the specimens so tested, no inhibitor was detected.

In every case the administration of the first dose of activator resulted in a fall in plasminogen level. The rate of fall was difficult to determine as specimens could not normally be obtained less than 18 hours after the first dose, but in every case the plasminogen level fell to zero or to very low values within 24 hours. In one case with an exteriorized Spitz-Holter valve where specimens could be obtained at frequent intervals, plasminogen fell to almost zero within 2 hours of administration of activator. In another such case serial specimens were obtained after a single dose and estimations of streptokinase and plasminogen were carried out. In this case there was some delay in the fall in plasminogen and the greatest fall occurred between 4 and 8 hours after the dose (Fig. 5). In the early cases when smaller doses were given, plasminogen fell to low levels after a dose but the fall was not so great as in later cases with

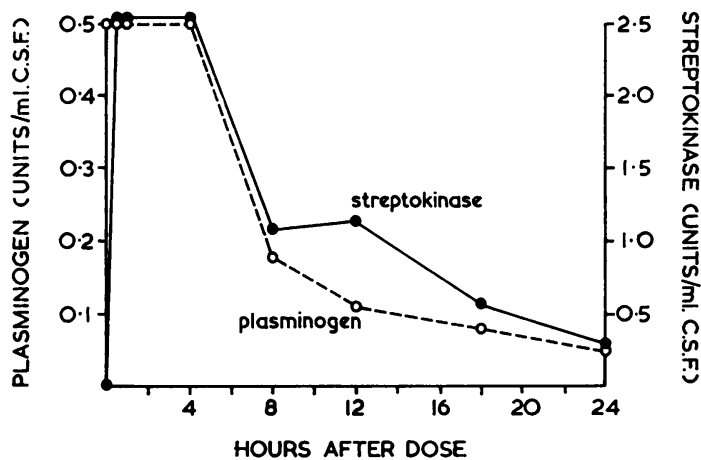

FIG. 5.-Changes in streptokinase and plasminogen levels in CSF after a single intraventricular dose of 25,000 units of streptokinase. 
higher dosage. In cases that received frequent doses the plasminogen levels remained low as long as treatment continued. At the end of a short course of treatment, plasminogen rose again, usually to its pre-treatment level, though in most cases levels remained low for at least 48 hours after the last dose and began to rise thereafter. In one case where treatment was discontinued and then started again, a rise in plasminogen occurred during the interval with a fall when treatment was restarted (Fig. 6).

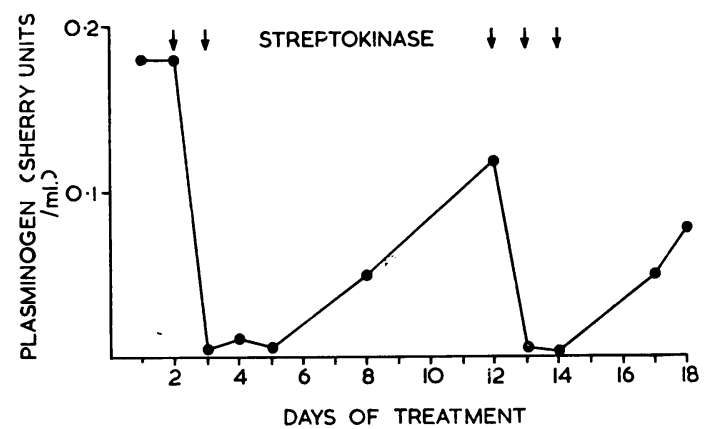

FIG. 6.-Plasminogen levels in cerebrospinal fluid of a patient treated with two courses of int ravent ricular streptokinase $(25,000$ units/dose).

Activator was demonstrated in a few of the specimens examined during treatment but could not be found in any specimen taken 24 hours after a single dose and was only detected 24 hours after the previous dose when several daily doses had already been given. The amounts found in these circumstances were of the order of 0.5 to $1 \%$ of the daily dose and appeared only in cases receiving large amounts (25,000 units daily or more).

Protein levels changed in a variable manner during treatment. In all cases protein was increased when treatment began, in a few to a very high level ( 2 to $3 \mathrm{~g} . / 100 \mathrm{ml}$.). No great increase was seen that could be attributed to the injections of activator. In some cases there was a steady fall throughout and following treatment (Cases 1, 2, 3, 8, 9, 10, 14, 16), in some the established rise continued (Cases 6, 7, 12, 15, 17), while in two others the results were irregular (Cases 5 and 11). In a few cases there was a halt in the rise while treatment was in progress followed by a resumption of the rise when treatment stopped (Cases 4 and 13).

Toxicity. In none of the cases treated was there any evidence of ill effect from the injections. The CSF cell count showed no adverse change and as a rule decreased as treatment continued. The EEG recordings taken continuously during and after the injections showed no abnormality attributable to the drugs except in a few cases a transient asymmetry. Clinical observation of the patients during injections revealed no sign of irritation of the CNS or of any distress attributable to the drugs.

\section{Clinical. These results are shown in Table 2 .}

Group I: Meningocele AND Meningitis (12 cases). Of the 12 babies with meningitis associated with a meningocele, 6 showed clinical improvement and 6 did not. Clinical improvement or a good result of treatment was judged by arrest of increasing head diameter, and in 5 of the 6 cases this occurred during treatment. In 2 cases the improvement was only temporary and hydrocephalus supervened later. In one case (Case 8) improvement was assumed on the basis of post-mortem findings. Though the patient died of hydrocephalus there was no fibrin deposition in the subarachnoid space and hydrocephalus appeared to be due to the developmental abnormality. In view of the findings at necropsy in cases of this kind not so treated, the absence of fibrin after a severe meningitis was taken to indicate some measure of success. Of the 12 cases 6 were treated with urokinase and 6 with streptokinase. Of the 6 urokinase cases 2 were improved clinically, while of the 6 streptokinase cases 4 were improved. Commencement of treatment was early in 4 of the 6 cases with a good result, but duration of treatment or dosage appeared to be unrelated to the result. Cases 1 and 5 died during treatment. Of the 10 cases that survived, only 4 were alive 6 months after treatment: 5 died of acute hydrocephalus and one of unknown cause at another hospital. Necropsies were carried out in 5 cases. In 4 of these the choroid plexus was buried in a dense fibrinous mass, collagenized in most cases. In all 5 there was hydrocephalus with Arnold-Chiari malformation: 3 showed fibrin deposition in the subarachnoid space. In Case 8 there was a non-patent aqueduct. No evidence was seen in any of the cases at necropsy of damage due to the ventricular taps, apart from small areas of old subarachnoid haemorrhage in 2 cases.

Group II: Meningitis With Subarachnoid BLock ( 2 cases). Two cases of subarachnoid block during the course of actue meningitis were treated. In both the immediate result was excellent, with disappearance of the block after the first and second injections respectively. Case 13 made poor progress due to brain damage from the meningitis and died later from bronchopneumonia. Abscesses were found in the cerebral hemispheres and the aqueduct was blocked by old inflammatory exudate. Case 14 made a good recovery and is well 5 months later: 
some bleeding occurred into the CSF after administration of urokinase intrathecally, but no excess of plasmin was demonstrated in the CSF or plasma, and the bleeding was considered to be traumatic, due to the repeated lumbar punctures.

Group III: INfection of SPITZ-Holter VAlve ( 2 cases). Two cases with infection of Spitz-Holter valves and ventriculitis were treated. In both, established hydrocephalus was already controlled by the atrio-ventricular shunt, but from past experience it was thought likely that bacterial colonization was accompanied by deposition of fibrin within the valve. In neither case was the infection cleared by combined intraventricular chemotherapeutic and fibrinolytic treatment, and both had the valves removed and changed. Case 15 later recovered and is well 1 year later. In Case 16, the new valve again became infected and the patient later died from bronchopneumonia.

Case 17, the 6-year-old child with the brain hernia, showed no improvement with treatment. Hydrocephalus persisted and she later died from untreated septicaemia due to Staphylococcus aureus. At necropsy the characteristic changes in the choroid plexuses were seen as in the other cases.

\section{Discussion}

In all the cases treated except Cases 13 and 14, congenital abnormalities were present predisposing to hydrocephalus. When meningitis occurs in such cases, necropsy reveals fibrin deposits, variable in extent, in the basal subarachnoid spaces and ventricles; the choroid plexus is often covered by a tough fibrinous deposit and in long-standing cases the lining of the ventricles is usually granular and may show fibrinous deposits as well. It seems likely, though difficult to prove, that deposition of fibrin in these sites might well contribute to the onset of hydrocephalus. In this event, fibrinolytic therapy might be expected to give improvement and, in 8 of the 17 cases treated, some measure of success has been achieved. In some this has been only temporary and surgical treatment has been necessary at a later date. It is difficult in the cases with congenital abnormalities to assess results categorically as the prognosis with or without infection is poor.

With regard to the effects of treatment on protein content in the CSF, plasmin is known to have proteolytic effects on substrates other than fibrin and fibrinogen, and it is possible that the fall in protein level seen in some of our cases might have been due to this effect. Again the results were variable, but the fall was greatest in those cases that received treatment for longer periods.
Reactions to intraventricular or intrathecal injections of streptokinase or urokinase were not seen in any of our cases. The highly purified preparations available at present appear to be free from such effects and are tolerated in large doses in intravascular thrombolytic therapy. Occasional batches, however, have been shown to be pyrogenic (McNicol, Douglas, and Bayley, 1962), and the results of such a reaction within the CNS might well be disastrous.

Antibodies were not detected in any of the cases where this test was made. In some, the treatment was given for too short a time for antibody formation to occur. In two cases treated for longer periods with streptokinase no antibody appeared and no reaction was noted to the later doses. Tillett and Sherry (1949) found that patients given intrapleural streptokinase developed specific anti-streptokinase in both pleural fluid and blood. We have examined the blood antistreptokinase titre in only one of our cases (Case 12), and no rise in titre was found after the course of streptokinase lasting 15 days. It is possible that with lower dosage and rapid local utilization of the streptokinase antibody formation might not be a problem in this type of treatment as it is recognized to be in intravascular therapy, though Tillett and Sherry also found that streptokinase given into the pleural cavity disappeared within a few hours of injection.

The series of cases described here is too small to permit comparison between streptokinase and urokinase; both activators are well tolerated and interact rapidly with plasminogen. Being of human origin, urokinase might be regarded as the more acceptable substance physiologically; some of our preparations showed traces of thromboplastic activity but this was probably unimportant in the presence of degenerative cerebral tissue.

\section{Summary}

The cerebrospinal fluid in health contains only traces of plasminogen but a rapid rise occurs with the onset of meningitis, whether bacterial or viral. This is most marked during the first few days but persists thereafter as long as the protein content remains high. Plasminogen in this form can be activated by streptokinase and urokinase to yield plasmin. With proper dosage of activator, utilization of plasminogen in the CSF is virtually complete.

Given intraventricularly or intrathecally, purified preparations of these activators are well tolerated and appear to induce a fibrinolytic state in the CSF. By this means early deposition of fibrin was probably prevented or cleared in 8 cases of meningitis and ventriculitis out of 17 treated.

Antibodies and inhibitors to these activators were 
not detected in the CSF even after repeated administration. Other factors influencing the response to treatment are discussed.

We are grateful to our colleagues, Dr. H. M. T. Coles, Mr. H. B. Eckstein, Mr. D. M. Forrest, and Mr. H. H. Nixon for the opportunity to treat patients under their care, and for their co-operation; we also acknowledge the help of Dr. L. Crome and Dr. M. Erdohazi for necropsies and of junior medical staff and nursing staff, in particular Sister M. Perry; we are grateful to Leo Laboratories Ltd. for supplies of urokinase and to Messrs. Kabi for streptokinase.

\section{REFERENCES}

Alkjaersig, N., Fletcher, A. P., and Sherry, S. (1959). The mechanism of clot dissolution by plasmin. J. clin. Invest., 38, 1086.
Blombäck, B., and Blombäck, M. (1956). Purification of human and bovine fibrinogen. Ark. Kemi., 10, 415.

Cathie, I. A. B. (1949). Bacterial fibrinolysin, its possible therapeutic application in tuberculous meningitis. J. clin. Path., 2, 73.

Fletcher, A. P. (1954). The preparation of partially purified plasmin, and an account of its intrathecal use in patients. J. clin. Invest., 33, 1242.

McNicol, G. P., Douglas, A. S., and Bayley, C. (1962). Experience with streptokinase infusions. Lancet, 2, 1297.

Newman, R. L. (1964a). A method for detecting and estimating plasminogen in cerebrospinal fluid. J. clin. Path., 17, 313. (1964b). An instrument for recording the lysis time of fibrin clots. ibid., 17, 194.

(1965). Method for the detection and measurement of antibodies to streptokinase in the cerebrospinal fluid. ibid., 18, 256.

Sherry, S., Lindemeyer, R. I., Fletcher, A. P., and Alkjaersig, N. (1959). Studies on enhanced fibrinolytic activity in man. J. clin. Invest., 38, 810 .

Tillett, W. S., and Sherry, S. (1949). The effect in patients of streptococcal fibrinolysin (streptokinase) and streptococcal desoxyribonuclease on fibrinous, purulent, and sanguinous pleural exudations. ibid., 28, 173. 